\title{
Phase structure of metallothermally reduced deep-sea concretion
}

\author{
J. Kopeček ${ }^{1 *}$, F. Laufek², A. Michalcová ${ }^{3}$, L. Šulcová ${ }^{3}$ A. Tsepeleva ${ }^{3}$, E. Chmelíková ${ }^{3}$, D. Nováček ${ }^{3}$, N.H. Vu ${ }^{3}$, \\ P. Dvořák ${ }^{3}$ and P. Novák ${ }^{3}$ \\ ${ }^{1}$ FZU - Institute of Physics of the Czech Academy of Sciences, Praha, Czech Republic, \\ ${ }^{2}$ Czech Geological survey, Praha, Czech Republic, \\ ${ }^{3}$ University of Chemistry and Technology in Prague, Praha, Czech Republic \\ kopecek@fzu.cz
}

Metallothermally reduced deep-sea nodules from Clarion-Clipperton Zone (Pacific Ocean) are investigated in this study. The aim is to prepare "natural alloy", to construct as simply as possible way to reduce nodules into the usable alloy without wasting the energy, mainly to avoid the purification of individual metals. Here used nodules contain manganese as the dominant element, whereas iron, nickel and copper are other major constituents besides aluminium and silicon. The deepest investigated is the aluminothermic process [1], nevertheless the other reducing metals - titanium and silicon - are investigated too.

The nodules were reduced and annealed alloys at $700{ }^{\circ} \mathrm{C}$ with various excess of aluminium $(0 \%, 10 \%$, and $20 \%)$. Using XRD there were found three, five and eight phases, some of them not listed in databases. Some other minor phases as sulphide MnS were found using SEM with EBSD/EDS coupled detectors.

There is a couple of interesting points. The formation of main manganese rich phase, which develops from $\beta-\mathrm{Mn}_{66} \mathrm{Ni}_{20} \mathrm{Si}_{14}$ phase $\left(P 2_{1} 3\right.$ space group) at $0 \%$ of excess to $\beta$-Mn phase ( $P 4_{1} 32$ space group) at $10 \%$ of excess and to $\alpha$-Mn phase ( $I-43 m$ space group) at $20 \%$ of excess. The separation of $\mathrm{Mn}_{2} \mathrm{FeSi}$ and $\mathrm{Mn}_{2} \mathrm{FeAl}$ phases. Those phases were just recently confirmed experimentally to exist.

[1] Novák P., Nguyen H. V., Šulcová L., Kopeček J., Laufek F., Tsepeleva A., Dvořák P. \& Michalcová A. (2021) Materials $14,561$.

Keywords: deep-sea nodules, metal reduction, aluminothermy, EBSD, manganese

We acknowledge Czech Science Foundation project 20-15217S for support and CzechNanoLab Research Infrastructure (LM2018110) by MEYS CR for SEM infrastructure support. 